\title{
Multi-Modality Imaging in a Hybrid Setting Facilitates Transcatheter Closure of a Traumatic Ventricular Septal Defect
}

\author{
Tamim Nazif, Rebecca T. Hahn, Robert Sommer, Susheel K. Kodali, \\ Mathew R. Williams, Isaac George \\ Divisions of Interventional Cardiology and Cardiothoracic Surgery, Columbia University College of \\ Physicians and Surgeons, New York, USA \\ Email: ig2006@columbia.edu
}

Received August 4, 2013; revised September 4, 2013; accepted September 11, 2013

Copyright (C) 2013 Tamim Nazif et al. This is an open access article distributed under the Creative Commons Attribution License, which permits unrestricted use, distribution, and reproduction in any medium, provided the original work is properly cited.

\begin{abstract}
We present a case of transcatheter closure of a traumatic ventricular septal defect (VSD), in which simultaneous 3-dimensional (3D) transesophageal echocardiography (TEE) and angiography played a critical role in defect sizing, device selection, and procedural guidance.
\end{abstract}

Keywords: Ventricular Septal Defect; Transcatheter; Percutaneous

\section{Case Presentation}

A 17-year-old man with no significant medical history suffered multiple stab wounds to the abdomen and chest. Exploratory laparotomy revealed 600 milliliters of blood in the abdomen, but no obvious source of hemorrhage. Immediately afterward, he developed pulseless electrical activity that was felt to be due to cardiac tamponade. An emergent left thoracotomy was performed with decompression of the pericardium and repair of a right ventricular (RV) laceration. Transfusion was required with more than 10 units of packed red blood cells and other blood products. Post-operatively, physical examination was notable for a loud holosystolic murmur at the lower sternal border. Echocardiography revealed a traumatic VSD with left to right shunting, depressed RV function, and borderline left ventricular (LV) function. The patient was transferred to our institution for VSD repair.

On initial evaluation, the patient was deemed to be a poor candidate for cardiopulmonary bypass and surgical VSD repair due to ongoing sanguinous drainage from the abdomen. We therefore elected to proceed with transcatheter closure of the VSD in the hybrid operating room, with planned open conversion if unable to complete percutaneously. Internal jugular venous and femoral arterial access were obtained. Invasive hemodynamic assessment revealed a significant left to right shunt (Qp/ Qs 1.8), but no pulmonary hypertension. Left ventriculo- graphy confirmed the presence of a VSD with left to right shunting, but was inadequate to allow precise localization or sizing of the defect (Figure 1A). TEE clearly demonstrated the defect with left to right shunting, a peak velocity of $3.8 \mathrm{~m} / \mathrm{s}$, and Qp/Qs 1.7. Simultaneous multiplane imaging (Figure 1B) and real time 3D imaging (Figure 1C) allowed visualization of a funnelshaped, slit-like defect in the mid interventricular septum that was broadest on the RV aspect, measuring $1.1 \mathrm{~cm} \times 1.4$ $\mathrm{cm}$ and narrowing to $1.0 \mathrm{~cm} \times 0.4 \mathrm{~cm}$ on the $\mathrm{LV}$ side. The defect tunneled obliquely through the septum with a length of $1.1 \mathrm{~cm}$ and narrowest color Doppler jet of 1.0 $\mathrm{cm} \times 0.36 \mathrm{~cm}$. Based on the TEE imaging, we chose to use a $16 \mathrm{~mm}$ Amplatzer Post-Infarct (PI) Muscular VSD Occluder (St. Jude Medical, Minneapolis, MN) on a compassionate use basis.

Transcatheter closure of the VSD was performed under fluoroscopic and 3D-TEE guidance. The VSD was successfully crossed by a retrograde transarterial approach with a Terumo angled glidewire (Terumo Medical, Somerset, NJ) and a Judkins right coronary catheter. Simultaneous angiography and multiplane and 3D TEE imaging was performed to guide wire positioning (Figure 2). The wire was then advanced to the left pulmonary artery, snared, and exteriorized via the internal jugular venous access to form a continuous arteriovenous loop. The Amplatzer septal occluder was delivered by the ante- 

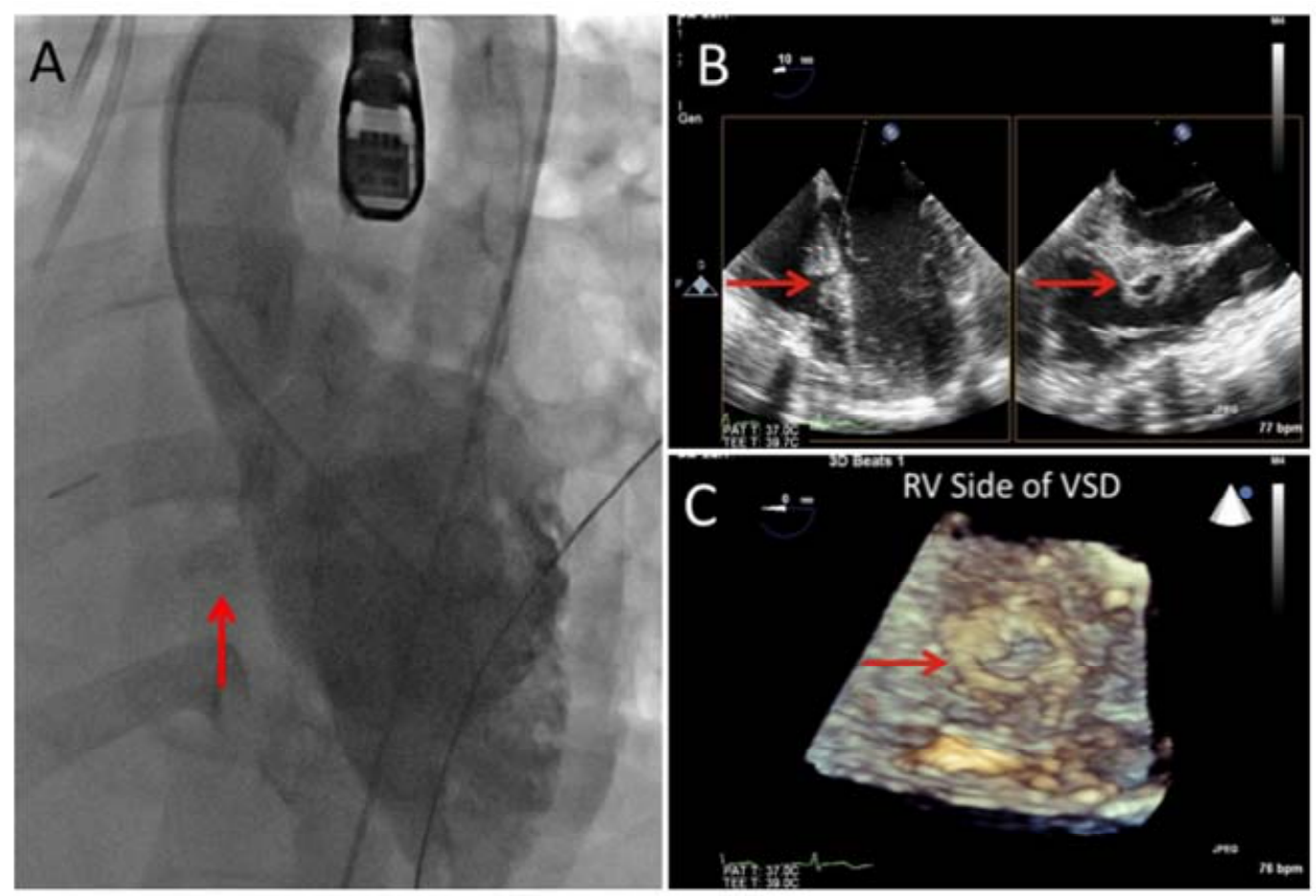

Figure 1. Imaging of the traumatic VSD. Panel A shows left ventriculography that demonstrates the VSD flow (arrow), but does not permit adequate localization or sizing of the defect. Panel B shows simultaneous multi-plane imaging of the VSD with the left image from the 4-chamber view and the right image in the orthogonal plane directed across the VSD (red arrow). Panel C is the user-defined 3D TEE reconstruction of the wide right ventricular aspect of the VSD (red arrow), which was the initial entry point of the knife into the septum.
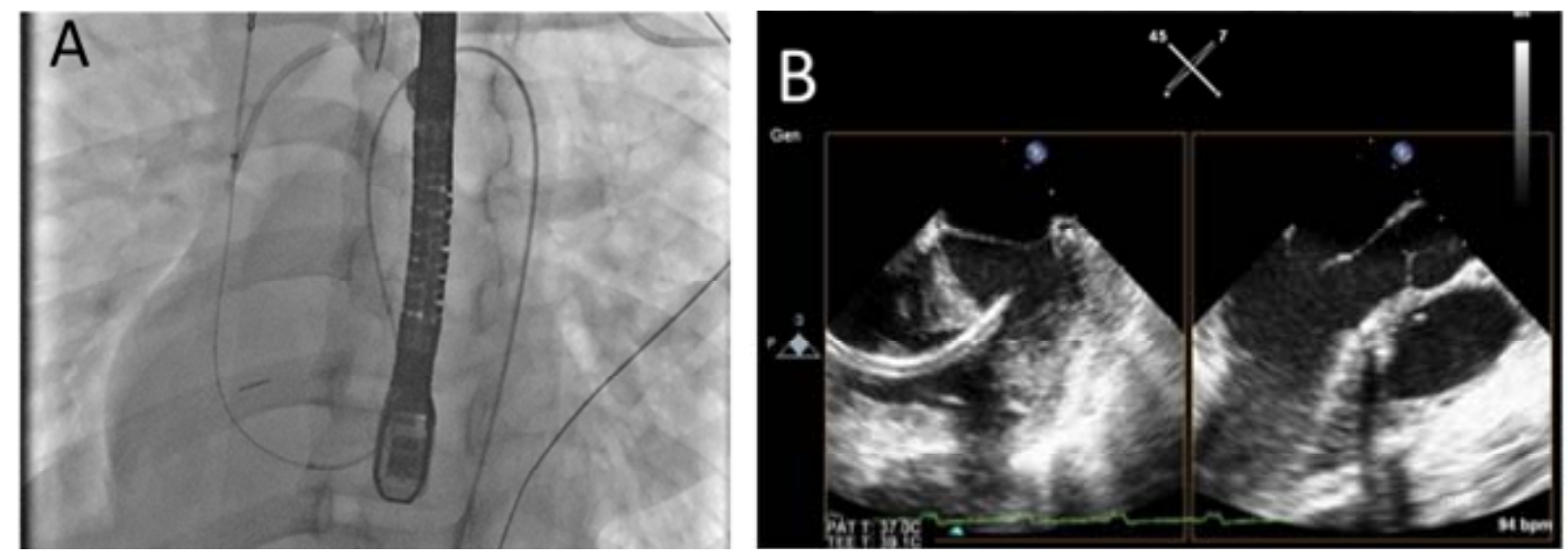

Figure 2. Imaging during intervention. Panel A shows fluoroscopic imaging of the wire across the VSD. Panel B shows simultaneous multiplane and 3D TEE imaging that was performed to guide and confirm wire (yellow arrow) positioning.

grade transvenous approach through a 9 French Amplatzer delivery sheath. Device positioning and adequacy of VSD closure was verified by angiography and 3DTEE (Figures 3A-C). The RV disk protruded slightly into the ventricular cavity, but 3D TEE imaging confirmed an intact tricuspid apparatus with no significant tricuspid regurgitation (Figure 3D). There was minimal residual shunt by color Doppler (Figure 3D), and the Qp/ Qs decreased to 1.1 by TEE and 1.2 by invasive assess- ment. The patient was discharged on post-operative day six and maintained on aspirin and clopidogrel for 6 months. He was seen in follow-up and reported return to his usual activities with no significant cardiovascular symptoms.

\section{Discussion}

Traumatic VSD is a rare, but important complication of penetrating cardiac trauma, blunt chest trauma, and car- 

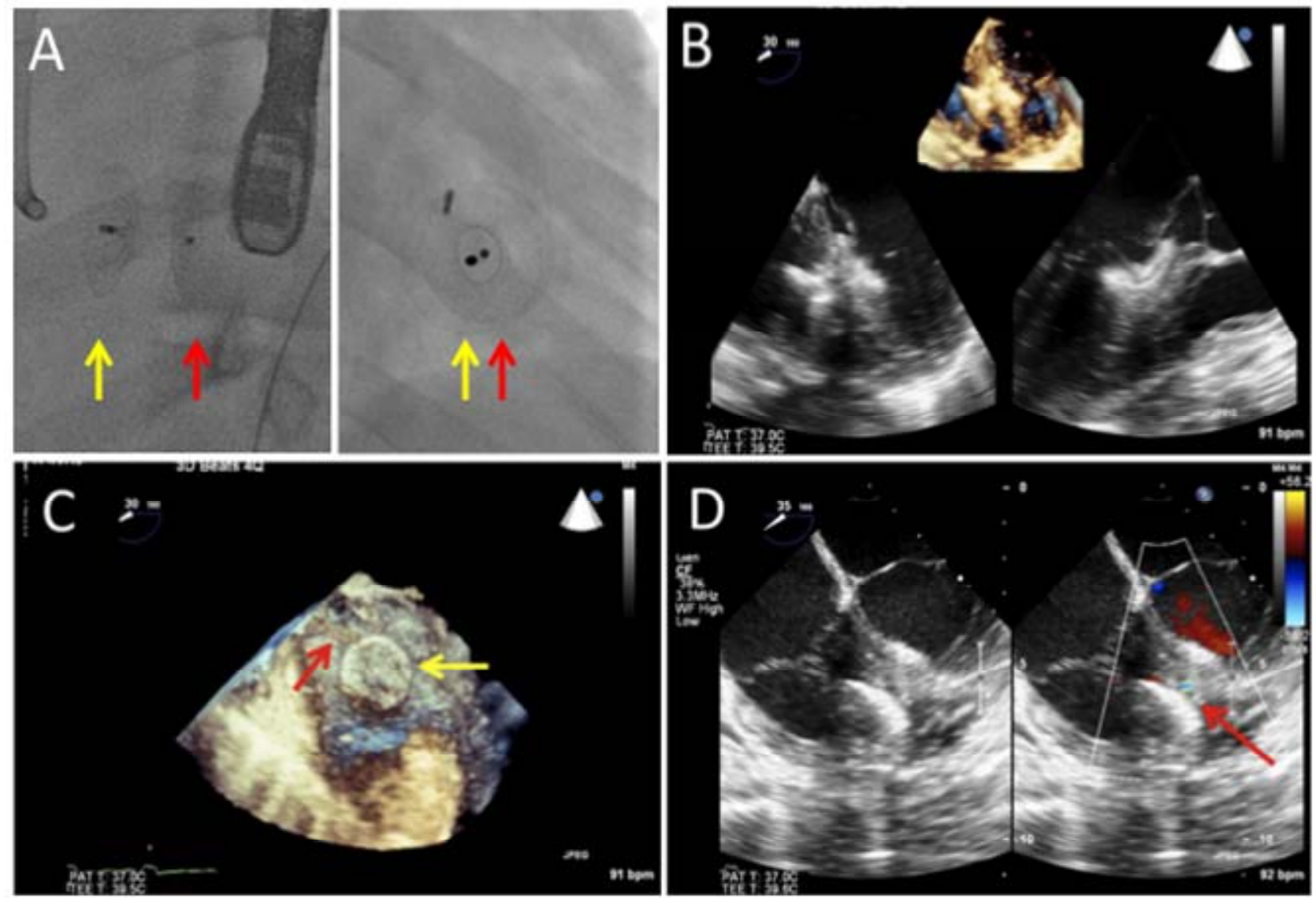

Figure 3. Imaging of the deployed Amplatzer PI Muscular VSD Occluder. Panel A is a fluoroscopic image of the deployed septal occluder. Panel B shows triplane imaging (3D volume at the top and simultaneous multiplane imaging in lower 2 images) of the device. Panel C shows a real-time 3D image of the RV disk (yellow arrow) protruded slightly into the ventricular cavity, but with an intact tricuspid apparatus (red arrow). Panel D shows a simultaneous two-dimensional and color Doppler image confirming minimal residual shunt (red arrow).

diac surgery. There is general consensus that defects with a significant shunt, represented by $\mathrm{Q}_{\mathrm{p}} / \mathrm{Q}_{\mathrm{s}}$ greater than 1.5 , should be repaired. Transcatheter closure of traumatic VSDs has been described in patients who are poor candidates for open surgical repair [1-4]. The technique of retrograde transarterial approach, creation of an arteriovenous loop, and antegrade transvenous delivery of a closure device has been well described. Successful closure of traumatic VSDs has been reported with an array of Amplatzer devices, including the atrial septal, membranous VSD, muscular VSD, PI muscular VSD, and duct occluders and the vascular plug $[3,4]$. We believe that the Amplatzer PI device used in the current case offers several potential advantages in this application. Given the normal myocardial thickness immediately adjacent to the slit-like VSD, the longer waist $(10 \mathrm{~mm})$ prevents possible translocation of the device due to inadequate straddling of the septum. In addition, the larger available sizes (16 to $24 \mathrm{~mm}$ waist diameter) allow oversizing of the device in defects that may be challenging to accurately size, without significantly flaring the RV disk.
In the context of the procedure described, 3D TEE imaging offers a number of advantages over two-dimensional imaging. Simultaneous multiplane and live 3D imaging allows accurate assessment of the morphology of irregularly shaped defects and therefore facilitates device selection. The addition of the elevational plane allows precise anatomic localization of thin wires and enhances imaging of the device during positioning and deployment. Finally, 3D TEE improves the assessment of possible complications such as device malposition, residual shunting, and tricuspid valve impingement. This case demonstrates the important role that careful preoperative planning and multi-modality imaging plays in the hybrid approach to traditionally surgical cardiac disease.

\section{REFERENCES}

[1] D. Pesenti-Rossi, F. Godart, A. Dubar and C. Rey, “Transcatheter Closure of Traumatic Ventricular Septal Defect: An Alternative to Surgery,” Chest, Vol. 123, No. 6, 2003, pp. 2144-2145. 
http://dx.doi.org/10.1378/chest.123.6.2144

[2] C. G. Cowley and R. E. Shaddy, "Transcatheter Treatment of a Large Traumatic Ventricular Septal Defect," Catheterization and Cardiovascular Interventions: Official Journal of the Society for Cardiac Angiography \& Interventions, Vol. 61, No. 1, 2004, pp. 144-146.

http://dx.doi.org/10.1002/ccd.10707

[3] C. Berry, W. S. Hillis and W. B. Knight, "Transcatheter Closure of a Ventricular Septal Defect Resulting from Knife Stabbing Using the Amplatzer Muscular VSD Oc- cluder," Catheterization and Cardiovascular Interventions: Official Journal of the Society for Cardiac Angiography \& Interventions, Vol. 68, No. 1, 2006, pp. 153-156. http://dx.doi.org/10.1002/ccd.20718

[4] S. Muyskens, J. Lasala and D. Balzer, "Percutaneous Closure of a Complex Posttraumatic Muscular Ventricular Septal Defect," Catheterization and Cardiovascular Interventions: Official Journal of the Society for Cardiac Angiography \& Interventions, Vol. 72, No. 3, 2008, pp. 408-412. http://dx.doi.org/10.1002/ccd.21659 